\title{
Fine needle aspiration cytology guided by ultrasound of suspicious axillary lymph nodes in breast cancer patients
}

Waldeir de Almeida Júnior ${ }^{1}$, Alexandre de Almeida Barra ${ }^{2,3 *}$, Henrique Moraes Salvador Silva ${ }^{1}$, Marco Antônio Dias Filho ${ }^{3}$, Henrique Lima Couto $^{4}$ and Breno Bernardes de Souza ${ }^{2}$

${ }^{1}$ Hospital Mater Dei Breast Unit Belo Horizonte, MG, Brazil

${ }^{2}$ Universidade Federal de Ouro Preto, Ouro Preto, MG, Brazil

${ }^{3}$ Instituto de Previdencia dos Servidores do Estado de Minas Gerais, Belo Horizonte, MG, Brazil

${ }^{4}$ REDIMAMA Reference Center for Breast Diagnosis, Brazil

\begin{abstract}
Purpose: The preoperative diagnosis of axillary status by means of Fine Needle Aspiration (FNA) guided by ultrasound is a method to obtain information on axillary lymph node status allowing decision about management of the axilla. This is a cross-sectional study aiming to analyze the use of ultrasound guided FNA for axillary lymph nodes and to determine the usefulness of this method in the preoperative staging of breast cancer patients candidates for sentinel lymph node biopsy (SNB).

Methods: Ultrasound guided FNA were performed in 41 patients who had presented abnormal alterations in axillary lymph nodes. Patients with malignant cytology avoided SNB and were submitted directly to axillary lymph node dissection (ALND), patients with negative cytology held SNB. The histological findings were compared with the results of ultrasound guided FNA.

Results: In evaluating FNA cytology, 18 (43.90\%) patients presented cytology compatible with malignancy and 23 (56.10\%) were benign. The axillary ultrasound guided FNA demonstrated a sensitivity of $81.8 \%$, specificity of $100 \%$, positive predictive value (PPV) of $100 \%$, negative predictive value (NPV) of $82.6 \%$ and $90,2 \%$ of accuracy.
\end{abstract}

Conclusion: The data demonstrate that the FNA is an effective procedure in preoperative assessment in patients with breast cancer.

\section{Introduction}

The status of axillary lymph nodes is an important prognostic factor of breast cancer with impact on the choice of treatment and survival of patients. In the absence of distant metastasis axillary status represents one of the most important prognostic factors in assessing the patient with breast cancer [1]. The evolution of surgical treatment of breast cancer, including in relation to axillary approach, contributed to the reduction of morbidity of treatment. The technique of sentinel lymph node biopsy (SNB) became increasingly common practice replacing the complete axillary lymph node dissection (ALND) in evaluation of axillary lymph nodes [2]. Different methods are used to detect metastases in axillary lymph nodes preoperatively $[3,4]$. The preoperative diagnosis of axillary status with Fine Needle Aspiration Cytology (FNA) guided by ultrasound is a method to obtain information on axillary lymph node status allowing decision about management of the axilla.

Recently, the field of breast cancer surgery has seen a dramatic shift with the results of the American College of Surgeons Oncology Group Z0011 trial [2]. This study, which randomly assigned patients with sentinel lymph node (SLN) positive breast cancer to SNB and axillar lymph node dissection (ALND) or to SNB alone, reported that ALND was not associated with any survival benefit and that both groups had an extremely low regional recurrence rate $(0.9 \%$ for $\mathrm{SNB}$ alone and 0.5 $\%$ for (ALND). Pre-operative ultrasound of the axilla is an extremely useful staging test as nodal disease identified by ultrasound and FNA biopsy is strongly correlated with tumour burden and the number of involved nodes $[5,6]$.

Indeed, by objectively documenting the presence of metastatic carcinoma by preoperative FNA, many patients can be directed toward full lymph node dissection, or to other management procedures of the axilla involved such as the neoadjuvant chemotherapy setting followed by SNB and axillary radiotherapy [7]. In the absence of metastatic carcinoma by the FNA, the patients can be submitted of SNB or omission of axillary surgery in randomized trials [8].

Currently, no imaging method has enough negative predictive value to avoid a surgical approach to the axilla in cases where no lymph node involvement is identified [9], however an increasing number of studies include such methods as part of the therapeutic planning. A study is currently being undertaken at the European Oncology Institute, comparing SLNB versus observation alone when axillary ultrasonography is negative in patients with small breast cancer candidates to breast conserving surgery [8].

${ }^{\star}$ Correspondence to: Alexandre de Almeida Barra, Universidade Federal de Ouro Preto, Ouro Preto, MG, Brazil, E-mail: barraalexandre@hotmail.com

Key words: breast cancer, ultrasound axillar lymph node, fine needle aspiration

Received: September 03, 2018; Accepted: September 14, 2018; Published: September 17, 2018 
FNA of axillary lymph nodes is a simple, minimally invasive technique that can be utilized as a tool to preoperative diagnosis of axilla. The objective of the current study was to determine the accuracy of FNA guided by ultrasound in detecting metastatic breast carcinoma in axillary lymph nodes.

\section{Materials and Methods}

This is a cross sectional study aiming to analyze the use of ultrasound guided FNA for axillary lymph nodes and to determine the usefulness of this method in the preoperative staging of breast cancer patients.

Axillary ultrasound was performed using a high frequency (10 $\mathrm{MHz}$ ) linear-array transducer.

The patient was lying down in a supine oblique position, with her hand above her head with the arm abducted and externally rotated ("bathing beauty" position). All findings was documented in orthogonal planes and a lesion's dimension was recorded. Colour Doppler ultrasound was used, it was with low wall filter settings and low velocity settings (higher pulse repetition frequency) to detect abnormal blood flow.

All the patients entered in the study were subjected to preoperative ultrasound evaluation of the axilla to determine the presence of lymph nodes that were sonographically suspicious of malignancy. Usually, a benign lymph node is ovoid, with a hypoechogenic cortex, extremely thin, less than $3 \mathrm{~mm}$ or even invisible at ultrasonography with a hyperechogenic hilum due to connective tissue trabeculae, lymphatic tissue cords and medullary sinusoids [10]. Doppler analysis shows vascularization in the hilum exclusively. Changes such as cortical thickening, hilum decrease or absence, changes in shape or vascular pattern, and size greater than $10 \mathrm{~mm}$ were considered suspicious [10]. Was included size that it used to be considered a criterion, although studies have not demonstrated a significant relationship between size and malignancy [11-13].

Written informed consent was obtained from the patients, and they were assured that their treatment would not differ in any way from the preexisting standard of care in the institution.

Ultrasound guided FNA was performed on 41 breast cancer patients presenting suspicious lymph nodes from March 2010 to February 2011. FNA was performed with a 23-gauge hypodermic needle on a syringe with vacuum application. For each lesion two to four passes were made in accordance with recommendations by the American Consensus Meeting on breast needle procedures [14].

Under real-time visualization, the needle was directed into the lymph node. The position of the needle within the lymph node at the time of FNA was documented on film. Direct smears from the aspirate were fixed in alcohol. Results of FNA stained by Hematoxylin Eosin (HE) were reported as malignant, benign, or insufficient material. Therefore, the FNAs remained with histologic follow-up, with SNB in FNA benign or full axillary lymph node dissection in FNA malignant. After surgical excision by SNB or ALND the lymph node were reported as malignant or benign. Sensitivity, specificity, positive predictive value (PPV) and negative predictive value (NPV) were applied to FNA using the final surgical histopathology results as gold standard.

Histopathologic examination of the sentinel node and other lymph nodes of the axilla, were performed separately. All nodes embedded in paraffin and cut in sections of $4 \mathrm{~mm}$ thickness, and stained by HE. One section of each node was examined. In cases where neoplastic cells were identified in the sentinel node, no further sections were made. In all the other cases, serial cuts were made at every $50 \mathrm{~mm}$ with thickness also of $4 \mathrm{~mm}$, five to six laminae stained by HE were thereby obtained for each sentinel node.

\section{Results} years).

The Median age of 41 patients was 54,8 years (range, 35 to 80

The histologic characteristics of the primary breast carcinomas included 32 cases of ductal invasive carcinoma (78\%), three cases of invasive lobular carcinoma, three cases of mixed invasive ductal and lobular carcinoma and one case of mucinous, tubular, and papillary carcinoma.

The median size of the primary tumor was $1.8 \mathrm{~cm}$ (range $0.4-3.5$ $\mathrm{cm})$. The median size of the punctioned axillary lymph nodes was $1.56 \mathrm{~cm}$ (range, $0.8-2.5 \mathrm{~cm}$ ) (Table 1). According to the Bloom-ScarffRichardson grading for breast carcinoma, there were 14 cases of grade 3 (43.1\%), 16 cases of grade 2 (44.8\%), and 11 cases of grade $1(12.0 \%)$ (Table 1). Despite having to rely on 'suspicious' imaging features, the more frequent is s lymph node size $>10 \mathrm{~mm}(92,68 \%)$, the cortical thickening (Figure 1) and abnormal fatty hilum (Table 2) (Figures 2 and 3).

The results of FNAC identified 18 malignant cases and benign cases summed 23. The final surgical histopatology results of lymph node identified 22 (54\%) malignant cases and 19 benign (46\%). There were no false-positive or inadequate results of FNAC. In the 23 benign FNAC, 19 were confirmed histologically. In the 4 false-negative cases, micrometastases were detected in 2 cases (Table 3).

Proportionally, there was a greater detection of axillary lymph nodes with cytology malignancy in tumors with histological grade III (12 affected in 17, 71\%), nuclear grade 3 (12 affected in 14, 86\%), lymphovascular invasion positive (14 affected at $20,70 \%)$, larger tumor size $>3 \mathrm{~cm}$ (10 affected at 14, 71,5\%). No significant complications of FNA were encountered in this series.

The sensitivity and specificity of the axillary lymph node FNA in all the cases (including both sentinel and full lymph node dissection

Table 1. Tumor and lymph node characteristic

\begin{tabular}{|l|c|c|c|}
\hline Characteristic & Axila & Axila- & Total \\
\hline Histological grade & 3 & 9 & 12 \\
\hline I & 7 & 5 & 12 \\
\hline II & 12 & 5 & 17 \\
\hline III & 14 & 6 & 20 \\
\hline Lymphovascular invasion & 8 & 7 & 15 \\
\hline Present & 0 & 6 & 6 \\
\hline Absent & & & \\
\hline Indeterminate & 9 & 4 & 13 \\
\hline Peritumoral inflammatory infiltrate & 13 & 10 & 23 \\
\hline Present & 0 & 5 & 5 \\
\hline Absent & & & \\
\hline Indeterminate & 1 & 2 & 3 \\
\hline Tumor size cm & 0 & 4 & 4 \\
\hline$<0.5$ & 11 & 8 & 19 \\
\hline $0.5-1.0$ & 10 & 4 & 14 \\
\hline $1.1-2.0$ & 0 & 1 & 1 \\
\hline $2.1-3.0$ & & & \\
\hline$>3.0$ & 11 & 0 & 2 \\
\hline Lymph node size cm & 9 & 10 & 19 \\
\hline$<1.0$ & & 9 & 20 \\
\hline $1.1-2.0$ & & \\
\hline$>2.0$ & & & \\
\hline
\end{tabular}




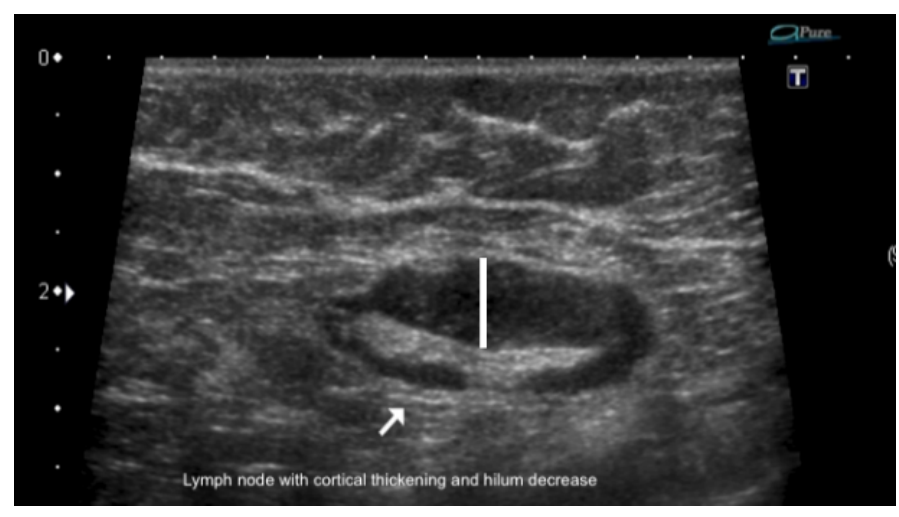

Figure 1. Lymph node with cortical thickening and hilum decrease

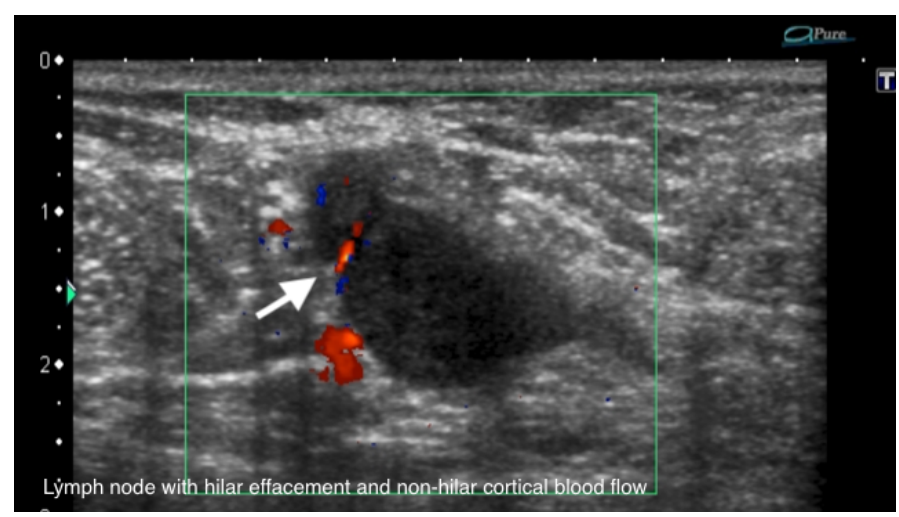

Figure 2. Lymph node with hilar effacement and non-hilar cortical blood flow

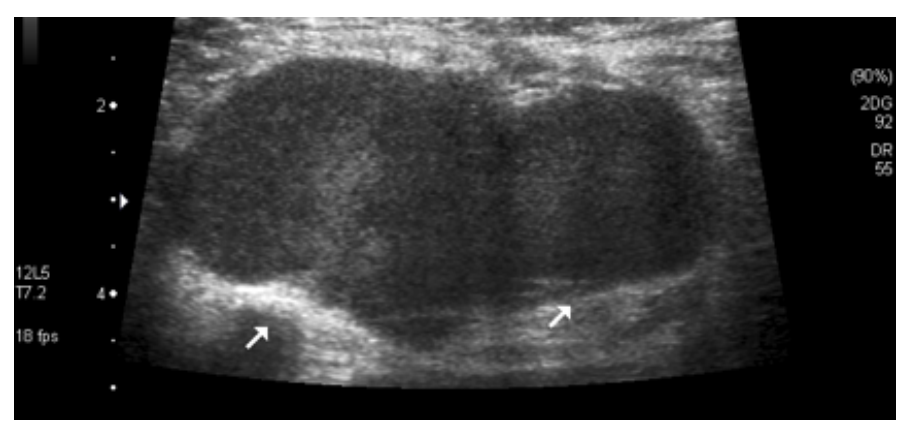

Figure 3. Lymph node with loss of the normal fatty hilum

Table 2. Suspicious characteristic of lymph node

\begin{tabular}{|c|c|c|c|}
\hline & Axila + & Axila - & Total \\
\hline Lymph node size $>10 \mathrm{~mm}$ & 19 & 19 & 38 \\
\hline Abnormal Fatty hilum & 15 & 04 & 19 \\
Cortical thickening & 18 & 01 & 19 \\
High vascular & 12 & 03 & 15 \\
Hypoechoic internal echo & 07 & 04 & 11 \\
\hline
\end{tabular}

Table 3. Accurancy of Fine need aspiration cytology of axillary lymph nodes by definitive lymph node histopathology exam

\begin{tabular}{|l|l|l|l|l|}
\hline FNAC & $\begin{array}{l}\text { Histopathology } \\
\text { Malignan }\end{array}$ & Exam Benign & Total & Percentagem \\
\hline $\begin{array}{l}\text { Malignan } \\
\text { Benign }\end{array}$ & 18 & 0 & 18 & 71.2 \\
\hline Total & 4 & 19 & 23 & 20 \\
\hline
\end{tabular}

*FNAC: Fine need aspiration cytology.

Sensitivity: $81,82 \% 95 \%$ CI $(59,72$ to $94,81 \%)$.

Especificity: $100 \% 95 \%$ CI $(82,35$ to $100,00 \%)$

Positive predictive value: $100 \% 95 \%$ CI $(81,47$ to $100,00 \%)$.

Positive predictive value: $82,61 \% 95 \%$ CI $(61,22$ to $95,05 \%)$. cases) were $81,82 \% 95 \%$ CI $(59,72$ to $94,81 \%)$ and $100 \% 95 \%$ CI $(82,35$ to $100,00 \%$ ), respectively. The PPV of the axillary lymph node FNA in all the cases (including both sentinel and full lymph node dissection cases) was $100 \% 95 \%$ CI $(81,47$ to $100,00 \%)$ and the NPV was $82,61 \%$ $95 \%$ CI $(61,22$ to $95,05 \%)$ and with an overall diagnostic accuracy of $90,2 \%$ (Table 3 ).

\section{Discussion}

Staging of axillary lymph nodes in invasive breast cancer is an important prognostic indicator. The sentinel lymph node biopsy is currently a standard procedure in staging axillary lymph node status. Alternatively, axillary staging can be done by ultrasound guided lymph node biopsy. As yet, if the axilla is proven to be positive for metastasis a complete axillary lymph node dissection (ALND) will be performed [15]. However, the results of the ACOSOG Z0011 trial showed that ALND may be redundant in selected patients with a positive sentinel node biopsy. Thus, the question arises whether the conclusions of the Z0011 trial can also be applied to patients found positive after ultrasound, since this population might differ from the sentinel node positive patients $[15,16]$.

The results suggest that patients whose axilla are staged positive by ultrasound represent a different population compared to those with a positive sentinel node. Based on these results, we state that the conclusions of the ACOSOG Z0011 trial cannot simply be implemented to the ultrasound positive selected patients. Thus, the role of axillary staging by ultrasound should be redefined since it might play an important role in selecting patients with extensive nodal involvement who may still benefit from axillary treatment with neoadjuvant chemotherapy or ALND [17].

Neoadjuvant chemotherapy induces rearrangements in nodal structure that may decrease the identification rate and therefore increase the rate of false-negatives for sentinel nodes at an estimated 11.5\% [18]. In all these situations, imaging can play a key role in assessing nodal status and can provide improved guidance for sentinel node indications [19]. As determination of the axillary lymph node status greatly affects the decision-making for the most appropriate lymph node dissection, it is critical to know the accuracy of the different techniques used in the preoperative assessment of the axillary lymph node status [15].

The results of FNA guided by ultrasound in establishing the diagnosis of axillary lymph nodes has wide variability depends on the size and location of the lymph node, and on the operator's and cytologist's experience. Several studies showed heterogeneous results, that may be influenced by patient selection, differences in biopsy technique, operator expertise, and interpretation of the results [15].

The data from the current study confirm that ultrasound-guided FNA cytology is a useful procedure with which to detect axillary lymph node metastases in breast carcinoma. Indeed, we found that the overall sensitivity and specificity for axillary lymph node FNAs in all our cases were $81 \%$ and $100 \%$, respectively, whereas the PPV was $100 \%$ and the NPV was $82 \%$ and overall diagnostic accuracy of $90,2 \%$. These results compare favorably with those in the literature, in which the sensitivity of ultrasound-guided FNAs for lymph nodes ranges from $36 \%$ to $86.4 \%$, the specificity ranges from $95.7 \%$ to $100 \%$, the PPV ranges from $92 \%$ to $100 \%$, and the NPV ranges from $67 \%$ to $70 \%$ [20-23].

The results indicate that the predictive power of a positive result is excellent, but as expected, a negative result is less helpful. The common causes of discrepancy between the initial and the final axillar lymph node staging are the failure to see the lymph nodes during ultrasound 
examination of the axilla, a small number of lymph nodes positive for metastases and small-sized metastases go unnoticed [24].

In the 23 benign FNA, 19 were confirmed histologically. In the 4 false-negative cases, micrometastases were detected in 2 cases (tumour deposit equal to or less than $2 \mathrm{~mm}$ ). Small deposits pose a difficulty with FNA sampling as the likelihood of erroneous sampling is high. Methods to improve the accuracy of sampling using ultrasound guided FNA would therefore be of benefit.

Rapid on-site evaluation, or ROSE, is a service that pathologists and cytotechnologists commonly perform to check the cellular content and adequacy of fine-needle aspiration smears. Material from the aspirate was assessed 'on site' in the ultrasound department by a cytopathologist or trained medical scientist in order to ensure the material taken was representative of the lesion sampled [25]. The introduction of ROSE ensured the efficiency of the FNA technique and conferred a higher rate of positive cytology [25]. In this 2 cases with micrometastases small deposits pose a difficulty with FNA sampling as the likelihood of erroneous sampling is high. Sometimes the false negative rate do not fall after ROSE had been introduced. Thus it appears that FNA guided by ultrasound is limited in the detection of axillary lymph nodes with a very low disease burden. The identification of small metastatic foci by ultrasound likely represents a fundamental limitation of US-FNA, regardless of operator or technique [26].

Minimum lymph node involvement, with deposits between 0.2 and $2 \mathrm{~mm}$ (micrometastasis), and $<0.2 \mathrm{~mm}$ (isolated tumor cells) is not related to significant morphological changes in the lymph node, thus limiting the usefulness of ultrasonography in such cases, so the diagnosis is made. The great challenge in sonographic diagnosis lies in the evaluation of lymph nodes whose cortex and hilum are observed in varied forms, corresponding to early stages of metastatic disease, with such cases being responsible for the largest proportion of false-negative cases $[27,28]$.

Metastatic cells in the lymph reach the lymph nodes through afferent lymphatic vessels on the convex aspect of the organ. Then, the lymph is filtered through the cortex, paracortex and finally the hilum. Metastatic deposits accumulate in the lymph node peripheral area, causing enlargement of the cortex, usually focal (at early stages), or uniform. The data from the current study showed that one of the most detectable alteration of the lymph node was cortical thickening (82\%) (Figure 1). A cortical thickness of $\geq 3 \mathrm{~mm}$ has been shown to be the most useful predictor of malignancy in clinical practice [29]. A focal cortical bulge or thickening is considered the earliest detectable morphologic change in the presence of metastasis, but this criterion is difficult to apply and has a low positive predictive value because it is non-specific. This finding is therefore considered indeterminate. A true abnormal cortical bulge is seen as focal thickening of the cortex that does not follow the margin of the echogenic hilum and should be distinctly hypoechoic, and this sign is more accurate if associated with another finding such as the presence of cortical in addition to hilar blood flow [29] (Figure 2).

According to literature no known biologic tumor marker to date can predict axillary lymph node metastases preoperatively. Different methods have been used, including palpation, ultrasonography alone, and ultrasonography combined with FNA or core needle biopsy. Clinical examination alone has been shown to be inaccurate, with a sensitivity ranging from $45.4 \%$ to $68 \%$ [24].

Similar to clinical examination, the accuracy of evaluation by ultrasound only also has been shown to be variable [24]. In this current study, the more frequent 'suspicious' imaging features are lymph node size $>10 \mathrm{~mm}(92,68 \%)$, cortical thickening and abnormal fatty hilum (Table 2). The characterization of axillary lymph nodes by sonography has also evolved in recent years. Morphologic criteria, such as cortical thickening, hilar effacement and non-hilar cortical blood flow, are more important than size criteria in the identification of metastases because enlarged axillar lymph node may be secondary to non malignant situations such as reactive hyperplasia, HIV/immunocompromised state, sarcoidose or other granulomatous diseases [29]. A cortical thickness of $\geq 3 \mathrm{~mm}$ has been shown to be the most useful predictor of malignancy in clinical practice [30].

A combination of these features has been used to assign the level of suspicion for metastatic disease by axillary ultrasound, although the size of the axillary lymph nodes has limited utility for determining the likelihood of metastatic disease [31].

Relying on 'suspicious' imaging features, the reported sensitivity of the evaluation of axillary lymph node status by ultrasound alone has ranged from $35 \%$ to $82 \%$, whereas its specificity has ranged from $73 \%$ to 97.9\% [30]. For ultrasound alone, Freitas, et al [30] quoted a PPV of $92 \%$, an NPV of $49 \%$, and a total accuracy of $67 \%$. Therefore, it appears that ultrasonography is not sufficiently accurate to replace histologic examination of the lymph nodes for the reliable determination of metastatic status.

As there is an overlap between the appearance of lymph nodes containing metastatic disease, normal hyperplastic lymph nodes, and reactive lymph nodes, preoperative tissue sampling under sonographic guidance is required to definitively diagnose axillary lymph node disease. Preoperative sampling of suspicious lymph nodes detected with imaging is almost always performed under sonographic guidance [15-17]. Once an abnormal lymph node is found, one faces the question on which procedure would be more appropriate: FNA or core biopsy. Such a decision should be made taking into account various peculiar aspects of each method [13].

FNA is fast, with high sensitivity and specificity, besides being less invasive; on the other hand, it requires an experienced cytologist, a professional who is only available at a small number of institutions. The current study was performed without assess 'on site' by a cytopathologist, but no insufficient cytology material was found, and a trained cytopathologist verified the smears with a great discretion. For each lesion two to four passes were made with the objective of reducing the insufficient cytology material rate in accordance with recommendations by the American Consensus Meeting on breast needle procedures [14]. This study is clearly limited by the small sample size, and because of that, subgroup analysis such as tumor sizes and grades, could not be performed.

The Clinical Practice Guideline Update Committee of American Society of Clinical Oncology think about shift considers the current reported false-negative rate (FNR) of SNB after neoadjuvant chemotherapy (NAC) in patients who present with involved axillary nodes (from $10 \%$ to $30 \%$ ) unacceptable and will take into consideration any new information regarding this specific patient population, for which there is insufficient data [32,33]. Currently, for most patients with biopsy proven node-positive breast cancer who receive NAC, ALDN is recommended, regardless of their response to treatment; $22 \%$ to $35 \%$ of these patients will have no residual disease in the axilla (axillary pathologic complete response [pCR]) after NAC. With better selection of patients for NAC and improved effectiveness of targeted therapy, these rates are likely to increase. ALDN could potentially be 
avoided in a large number of node-positive patients if SNB was shown to be accurate at identifying those who have achieved an axillary pCR after NAC [26].

Fine Needle Aspiration Cytology (FNA) guided by ultrasound may be indicated after completion of neoadjuvant chemotherapy before surgery to evaluate for residual nodal disease in patients who initially present with node positive breast cancer. A prior single-institution study at The University of Texas MD Anderson Cancer Center showed that use of axillar ultrasound for patient selection decreases the FNR of SLN surgery. In an era where regional node irradiation is increasingly used, the relevance of leaving residual disease in the undissected axilla of patients after NAC is unknown and is currently being investigated [30].

By multivariable analysis, tumor size and grade were significantly associated with axillary recurrence. It was concluded that the high fiveyear survival and low cumulative incidence of axillary recurrence in this cohort provided justification for the increasingly common practice of foregoing ALND in women with minimal sentinel node involvement, and suggested that ALND can safely be avoided in women with small, low-grade tumors [34]. The data from the current study showed that there was a greater detection of axillary lymph nodes with cytology malignancy in tumors with histological grade III (12 affected in $17,71 \%)$, nuclear grade 3 (12 affected in 14,86\%), lymphovascular invasion positive (14 affected at $20,70 \%)$, larger tumor size $>3 \mathrm{~cm}(10$ affected at 14,71,5\%). This results are consistent with Maniero, et al. [31] who found that the sensitivity of ultrasound-guided FNA was $29 \%$ in patients with primary tumors $\leq 1 \mathrm{~cm}, 50 \%$ in patients with tumors $>1$ to $\leq 2 \mathrm{~cm}, 69 \%$ in patients with tumors $>2$ to $\leq 5 \mathrm{~cm}$, and $100 \%$ in patients with tumors $>5 \mathrm{~cm}$.

Nevertheless a subset of patients might be at high risk of developing overt axillary disease and efforts should be made to identify such patients by ancillary analyses of the results of ongoing or recently published clinical trials [25]. Notwithstanding the disappointing early closures of Z0011 and 23-01 trials, evidence from other sources appears sufficiently comprehensive to justify not performing ALND in women with small cancers having favorable prognostic factors [32,33].

Ultrasound-guided FNA is fast, inexpensive, not associated with any significant morbidity, and may be able to obtain information on axillary lymph node status allowing for decisions on management of the axilla. Research that allows enhanced application of ultrasound and ultrasound-guided needle biopsy to specifically identify and biopsy sentinel nodes and to discriminate between patients with minimal versus advanced nodal metastatic involvement is likely to have the most impact on future management of the axilla in patients newly diagnosed with breast cancer [15].

The identification of new prognostic markers, the better understanding of tumors behaviour and the technological developments in imaging methods have a great potential of bringing changes in axillary staging in the future, by selecting patients eligible to less aggressive interventions.

\section{Authorship}

Waldeir de Almeida Júnior, MD. Hospital Mater Dei Breast Unit, Belo Horizonte, MG, Brazil.

\section{Funding}

No Funding information

\section{Competing interest}

No Competing interest

\section{References}

1. Carter CL, Allen C, Henson DE (1989) Relation of tumor size, lymph node status and survival in 14.740 breast cancer cases. Cancer 63: 181-187.

2. Giuliano AE, Hunt KK, Ballman KV (2011) Axillary dissection vs no axillary dissection in women with invasive breast cancer and sentinel node metastasis: a randomized clinical trial. JAMA 305: 569-575.

3. Pamilo M, Soiva M, Lavast EM (1989) Real-time ultrasound, axillary mammography, and clinical examination in the detection of axillary lymph node cancer case in breast cancer patients. $J$ Ultrasound Med 8: 115-120.

4. Mainiero MB (2010) Regional lymph node staging in breast cancer: The increasing role of imaging and ultrasound-guided axillary lymph node fine needle aspiration. Radiol Clin North Am 48: 989-997.

5. Cools-Lartigue J, Meterissian S (2012) Accuracy of axillary ultrasound in the diagnosis of nodal metastasis in invasive breast cancer: a review. World J Surg 36: 46-54.

6. Cools-Lartigue J, Sinclair A, Trabulsi N (2013) Preoperative axillary ultrasound and fine-needle aspiration biopsy in the diagnosis of axillary metastases in patients with breast cancer: predictors of accuracy and future implications. Ann Surg Oncol 20: 819827.

7. Reimer T, Hartmann S, Stachs A (2014) Treatment of the Axilla in Early Breast Cancer: Concepts from the National Surgical Adjuvant Breast and Bowel Project B-04 to the Planned Intergroup Sentinel Mamma Trial. Breast care 9: 87-95.

8. Gentilini O, Veronesi U (2012) Abandoning sentinel lymph node biopsy in early breast cancer? A new trial in progress at the European Institute of Oncology of Milan (SOUND: Sentinel node vs Observation after axillary ultrasound) The Breast 21: 678681

9. Rahbar H, Partridge SC, Javid SH (2012) Imaging axillary lymph nodes in patients with newly diagnosed breast cancer. Curr Probl Diagn Radiol 41: 149-158.

10. Ecanow JS, Abe H, Newstead GM (2013) Axillary staging of breast cancer: what the radiologist should know. Radiographics 33: 1589-1612.

11. Stravos AT (2004) Evaluation of regional lymph nodes in breast cancer patients. In: Stravos AT (Ed) Breast US. Philadelphia: Lippincoatt pp. 834-876.

12. Popli MB, Sahoo M, Mehrotra N (2006) Preoperative ultrasound-guided fine-needle aspiration cytology for axillary staging in breast carcinoma. Australasian radiology 50: $122-126$.

13. Pinheiro DJPC, Elias S, Nazário ACP (2014) Axillary lymph nodes in breast cancer patient: sonographic evaluation. Radio Bras 47: 240-44.

14. Cytology sub-group of the National Co-ordinating Committee for Breast Screening Pathology (1992) Guidelines for cytology procedures and reporting in breast cancer screnning. NHSBSP Publications No. 22.

15. Houssami N, Diespstraten SCE, Cody HS (2014) Clinical utility of ultrasound-needle biopsy for preoperative staging of the axilla in invasive breast cancer. Anticancer Res 34: 1087-1097.

16. Verheuvel N, Van den Hoven I, Voogd AC (2014) The role of ultrasound guided lymph node biopsy in axillary staging of invasive breast cancer in the post-ACOSOG Z0011 trial era. Ann Surg Oncol 22: 409-415.

17. Ciatto S, Brancato B, Risso G (2007) Accuracy of fine needle aspiration cytology (FNAC) of axillary lymph nodes as a triage test in breast cancer staging. Breast Cancer Res Treat 103: 85-91.

18. Classe JM, Bordes V, Campion L (2009) Sentinel lymph node biopsy after neoadjuvant chemotherapy for advanced breast cancer: results of Ganglion Sentinelle et Chimiothérapie Néoadjuvante, a French prospective multicentric study. J Clin Oncol 27: 726-232.

19. Maxwell F, Margerie MC, Bricout M (2015) Diagnostic strategy for the assessment of axillar lymph node status in breast cancer. Diagn Interv Imaging 96: 1089-1101.

20. Bonnema J, van Geel AN, van Ooijen B (1997) Ultrasound-guided aspiration biopsy for detection of nonpalpable axillary node cancer cases in breast cancer patients: new diagnostic method. World J Surg 21: 270-274.

21. Davis JT, Brill YM, Simmons S (2006) Ultrasound-guided fine-needle aspiration of clinically negative lymph nodes versus cancer case node mapping in patients at high risk for axillary metastasis. Ann Surg Oncol 13: 1545-1552.

22. Ciatto S, Brancato B, Risso G (2007) Accuracy of fine needle aspiration cytology (FNAC) of axillary lymph nodes as a triage test in breast cancer staging. Breast Cancer Res Treat 103: 85-91. 
23. de Kanter AY, van Eijck CH, van Geel AN (1999) Multicentre study of ultrasonographically guided axillary node biopsy in patients with breast cancer. $\mathrm{Br} J$ Surg 86: 1459-1462.

24. Bedrosian I, Bedi D, Kuerer HM (2003) cancer clinicopathological factors on sensitivity of axillary ultrasonography in the detection of axillary nodal cancer case in patients with breast cancer. Ann Surg Oncol 10: 1025-30.

25. DP O'Leary, O O'Brien, N Relihan (2012) Rapid on-site evaluation of axillary fineneedle aspiration cytology in breast cancer. Br J Surg 99: 807-812.

26. Fung AD, Collins JA, Campassi C (2014) Performance Characteristics of UltrasoundGuided Fine-Needle Aspiration of Axillary Lymph Nodes for Metastatic Breast Cancer Employing Rapid On-Site Evaluation of Adequacy. Cancer Cytopathol 122: 282-291.

27. Cho N, Moon WK, Han W (2009) Preoperative sonographic classification of axillary lymph nodes in patients with breast cancer: node to node correlation with surgical histology and sentinel node biopsy results. AJR Am J Roentgenol 193: 1731-1737.

28. Abe H, Schmidt RA, Kulkarni K (2009) Axillary lymph nodes suspicious for breas cancer metastasis: sampling with US-guided 14- gauge core-needle biopsy clinical experience in 100 patients. Radiology 250: 41-49.
29. Dialani V, James DF, Slanetz PJ (2015) A practical approach to imaging the axilla. Insights Imaging 6: 217-229.

30. de Freitas R, Costa MV, Schneider SV (1991) Accuracy of ultrasound and clinical examination in the diagnosis of axillary lymph node cancer case in breast cancer. Eur J Surg Oncol 17: 240-244.

31. Mainiero MB, Cinelli CM, Koelliker SL (2010) Axillary Ultrasound and FineNeedle Aspiration in the Preoperative Evaluation of the Breast Cancer Patient: An Algorithm Based on Tumor Size and Lymph Node Appearance. American Journal of Roentgenology 195: 1261-1267.

32. Boileau JF, Poirier B, Basik M (2014) Sentinel Node Biopsy After Neoadjuvant Chemotherapy in Biopsy-Proven Node-Positive Breast Cancer: The SN FNAC Study. J Clin Oncol 33: 258-264.

33. Boughey JC, Ballman KV, Hunt KK (2015) Axillary Ultrasound After Neoadjuvant Chemotherapy and Its Impact on Sentinel Lymph Node Surgery: Results From the American College of Surgeons Oncology Group Z1071 Trial (Alliance). J Clin Oncol $33: 1-8$

34. Galimberti V, Chifu C, Perez SR (2011) Positive axillary sentinel lymph node: Is axillary dissection always necessary? The Breast 20: 96-98.

Copyright: (2018 Waldeir de Almeida J. This is an open-access article distributed under the terms of the Creative Commons Attribution License, which permits unrestricted use, distribution, and reproduction in any medium, provided the original author and source are credited. 